\title{
Scatter-free breast imaging using a monochromator coupled to a pixellated spectroscopic detector
}

\author{
F.H.Green ${ }^{1}$, M. C. Veale $^{2}$, M. D. Wilson ${ }^{2}$, P. Seller ${ }^{2}$, J. Scuffham $^{3}$, S.Pani $^{1}$ \\ ${ }^{1}$ Department of Physics, University of Surrey, Guildford, UK; ${ }^{2}$ STFC Rutherford Appleton \\ Laboratories, Didcot, UK; ${ }^{3}$ Department of Nuclear Medicine, Royal Surrey County Hospital, \\ Guildford, UK
}

\begin{abstract}
This project uses the combination of a spectroscopic detector and a monochromator to produce scatter free images for use in mammography.

Reducing scatter is vital in mammography, where typical structures have either low contrast or small dimensions. The typical method to reduce scatter is the anti-scatter grid, which has the drawback of absorbing a fraction of the primary beam as well as scattered radiation. An increase in the dose is then required in order to compensate.

Compton-scattered X-rays have lower energy than the primary beam. When using a monochromatic beam and a spectroscopic detector the scattered beam will appear at lower energies than the primary beam in the detected spectrum. Therefore if the spectrum of the detected X-rays is available, the scattered component can be windowed out of the spectrum, essentially producing a scatter free image.

The monochromator used in this study is made from a Highly Orientated Pyrolytic Graphite (HOPG) crystal with a mosaic spread of $0.4^{\circ} \pm 0.1^{\circ}$.

The detector is a pixellated spectroscopic detector that is made from a $2 \mathrm{~cm} \mathrm{x} 2 \mathrm{~cm} \times 0.1 \mathrm{~cm}$ CdTe crystal with a pixel pitch of $250 \mu \mathrm{m}$ and an energy resolution of $0.8 \mathrm{keV}$ at $59.5 \mathrm{keV}$.

This work presents the characterisation of the monochromator and initial imaging data. The work shows a contrast increase of $20 \%$ with the removal of the low energy Compton scattered X-rays.
\end{abstract}

Keywords: Monochromatic X-rays, Mammography, Scatter removal, Spectroscopic Detector

\section{INTRODUCTION}

Mammography is the imaging of breast tissue in order to detect features in the tissue that could suggest the presence of breast cancer. These features, such as calcification and masses, are small in size or low in contrast, respectively. This puts stringent requirements on the imaging system in terms of spatial and contrast resolution ${ }^{1,2}$. In addition, breast tissue is sensitive to radiation and therefore the dose to the patient needs to be kept at a minimum ${ }^{3}$.

One of the causes of image degradation in X-ray imaging is Compton scattering ${ }^{4}$. In Compton scattering, X-rays loose energy and change their direction. This reduces image quality by adding, in a first approximation, a uniform background that reduces scatter.

Current radiographic techniques reduce scatter using an anti-scatter grid. This is a lead grid that absorbs X-rays with a direction that is not parallel to the primary beam. This has the downside of absorbing a proportion of the primary beam and therefore an increase in the dose is required to compensate ${ }^{5}$. This project looks at an alternative method of scatter removal using a pixellated spectroscopic detector combined with a monochromator.

A spectroscopic detector allows windowing of the energy spectrum. Compton scattered X-rays loose energy in the scattering process and will appear at a lower energy than the primary beam. Therefore, they can be windowed from the primary spectrum and hence produce a virtually scatter free image.

Using a monochromator has the additional benefit of tuning the energy to suit the thickness of the breast tissue being imaged. 


\subsection{Experimental setup}

\section{MATERIALS AND METHODS}

The experimental setup (figure 1) consists of a Hamamatsu L6732-01 X-ray source with a tungsten anode operated at $35 \mathrm{keV}$, a $10 \mathrm{~mm}$ thick brass collimator with a $1 \mathrm{~mm}$ x $20 \mathrm{~mm}$ slit, a Highly Ordered Pyrolytic Graphite crystal combined with a goniometer, a test object and a HEXITEC pixellated spectroscopic detector.

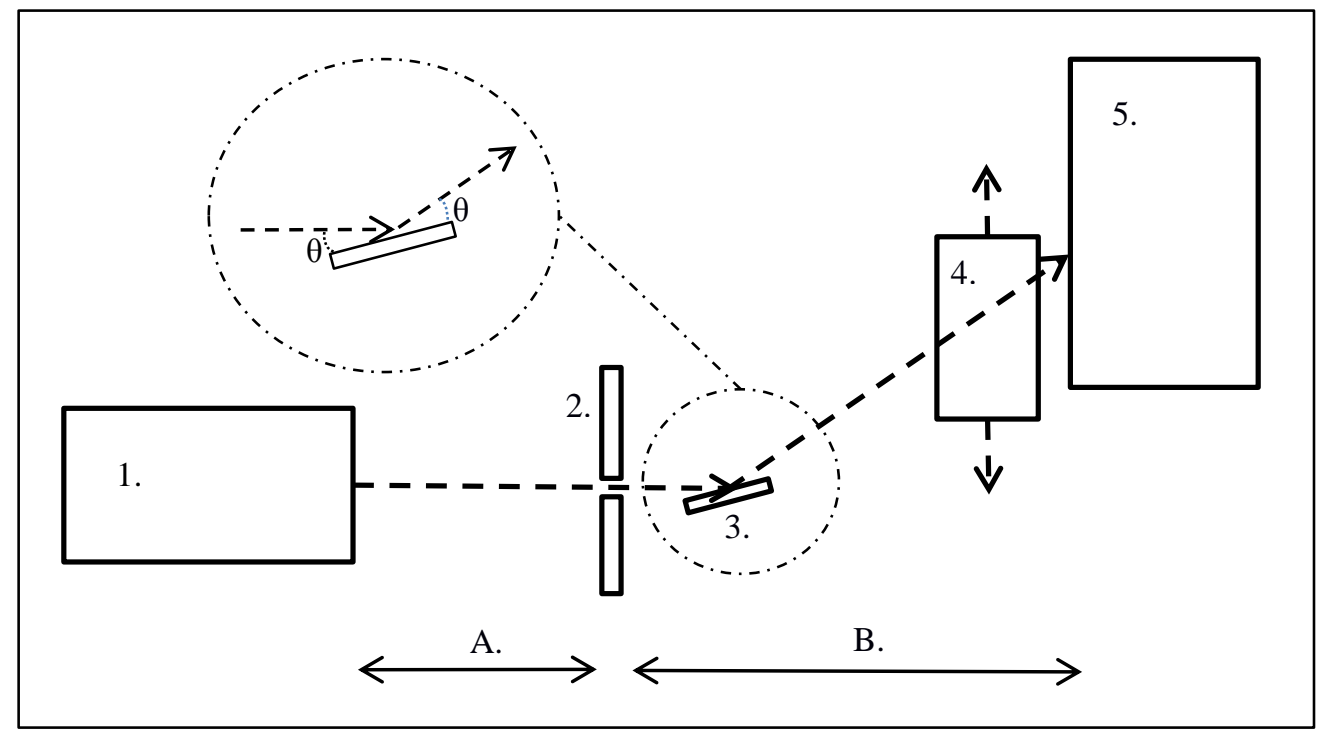

Figure 1. Diagram of the monochromator setup. On the diagram, 1 is the X-ray source, 2 is the collimator, 3 is the mosaic crystal, 4 is the test object and 5 is the HEXITEC spectroscopic detector. The angle for diffraction $\theta$ is shown. A is the source to collimator distance and is equal to $60 \mathrm{~cm} \mathrm{~B}$ is the collimator to Detector distance and is equal to $41 \mathrm{~cm}$.

\section{Monochromator}

The monochomator is a $2 \mathrm{~cm} \times 2 \mathrm{~cm} \times 0.2 \mathrm{~cm}$ Highly Orientated Graphite (HOPG) crystal with a mosaic spread of $(0.4 \pm$ $0.1)^{\circ}$ combined with a goniometer in order to adjust the angle of the crystal ${ }^{6}$. The relationship between the angle of the crystal and the energy of the monochromatic peak produced is given by Bragg's law (1). This states that for an X-ray beam incident on a crystal (lattice spacing d) with an angle $\theta$, only certain wavelengths $(\lambda)$ will constructively interfere with the crystal ${ }^{7}$.

$$
n \lambda=2 d \sin \theta
$$

For a single angle and crystal lattice spacing only integer multiples of the wavelength $\lambda$ will diffract from the crystal. This method has shown some promising results when combined with a synchrotron source, with an intensity several orders of magnitude greater than that of a conventional source. When using a conventional source, the intensity of the diffracted beam is not sufficient for imaging purposes. To solve this, a crystal with a mosaic spread will be used; this has a range of lattice orientations that will produce a small variation in the distribution of diffracted wavelengths and hence increase the overall intensity of the diffracted beam. This is demonstrated in figure 2 where the diffracted spectra are shown for both a perfect crystal and a mosaic crystal. 


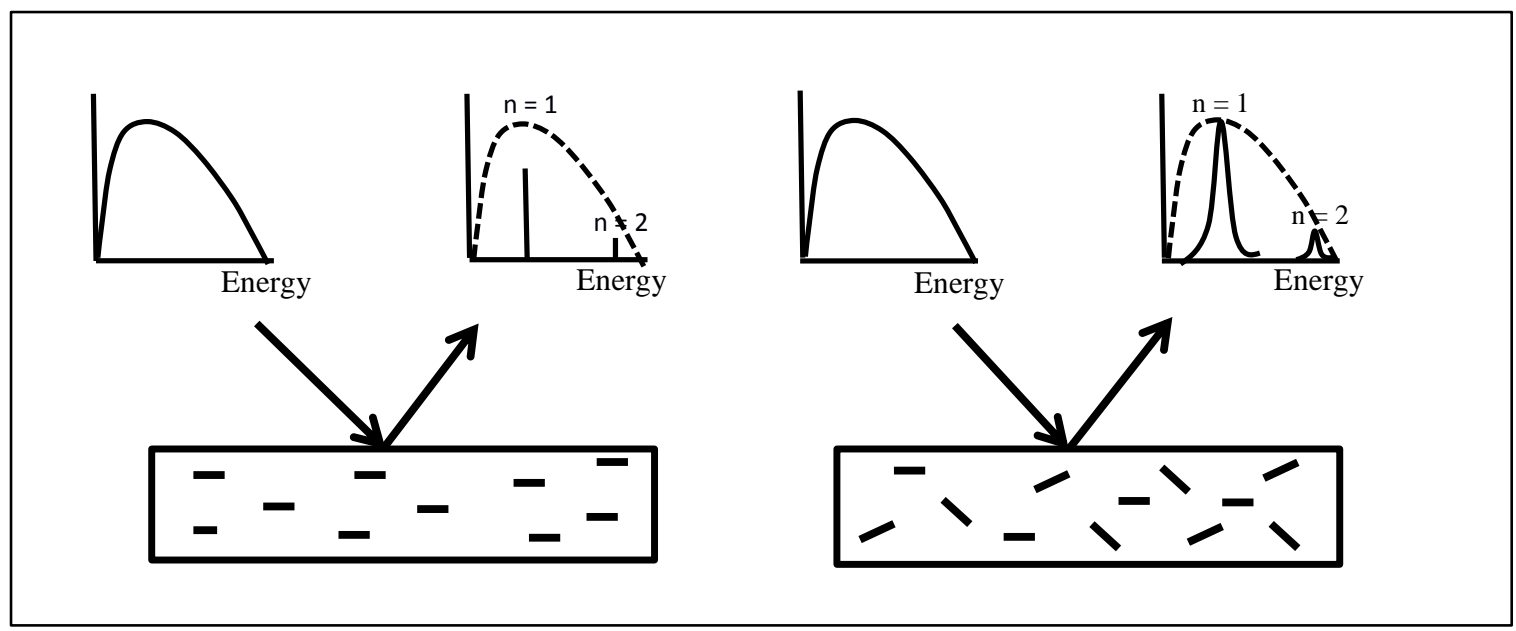

Figure 2. A diagram of the monochromatic peak produced with a crystal with a single orientation and with a crystal with a range of orientations.

\section{HEXITEC Pixellated Spectroscopic Detector}

The HEXITEC detector consists of an $80 \times 80$ array of pixels each producing an energy spectrum of the X-rays that are detected. The sensor is a $2 \mathrm{~cm} \times 2 \mathrm{~cm} \times 0.1 \mathrm{~cm}$ CdTe wafer; the readout array has a $250 \mu \mathrm{m}$ pixel pitch with $50 \mu \mathrm{m}$ interpixel spacing and an energy resolution of $0.8 \mathrm{keV}$ at $59.5 \mathrm{keV}^{8}$. Each channel has an identical set of electronics associated with it consisting of amplifiers, charge shapers and a peak hold circuit that measures the magnitude of the pulse produced by each photon when it interacts with the CdTe material, which can then be converted to energy by linear calibration ${ }^{9}$. The detector can also be cooled to improve the spectral resolution.

\section{Test object}

The test object consists of a $10 \mathrm{~cm}$ x $10 \mathrm{~cm}$ x $0.5 \mathrm{~cm}$ Perspex block with three $5 \mathrm{~mm}$ in diameter holes and three $2 \mathrm{~mm}$ in diameter holes in the middle. Two sets of holes are filled with Nylon and Wax inserts and the final set left empty. The Nylon and the wax have a similar attenuation coefficient to Perspex and hence produce low contrast when imaged. The test object includes a series of additional Perspex blocks of different widths so that the thickness of the overall test object can be varied to simulate different breast thicknesses.

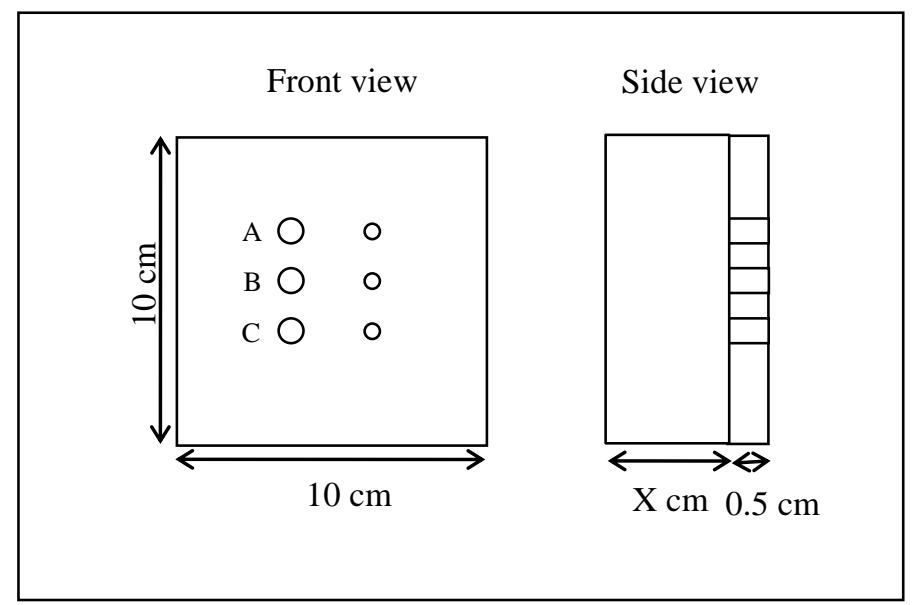

Figure 3. The front and side view of a Perspex test object. A is the Nylon detail, B is the wax detail and $\mathrm{C}$ is the air detail. Each detail is $0.5 \mathrm{~cm}$ thick and has two sizes, $0.5 \mathrm{~cm}$ and $0.2 \mathrm{~cm}$. The width of the Perspex with the embedded details is 0.5 $\mathrm{cm}$ thick. The width of the additional Perspex (width $\mathrm{X} \mathrm{cm}$ ) can be changed depending on the thickness of breast tissue that is being simulated. 


\subsection{Energy optimisation}

The optimal X-ray energy required to image a patient is dependent on the thickness of the breast being imaged. A thicker breast requires a higher energy of X-ray to produce an image with an adequate intensity. For the range of energies used in mammography, the higher the X-ray energy used, the higher the contrast but also the higher the dose to the patient ${ }^{10}$. Therefore there needs to be a compromise between image quality and dose.

To calculate the optimum monochromatic X-ray energy for each thickness of test object the image quality factor (IQF) (2) was calculated within the range of energies produced by the monochromator for a range of Perspex thicknesses ${ }^{11}$.

$$
I Q F=\frac{S N R^{2}}{D o s e}
$$

The Signal to noise ratio (SNR) is given by equation (3) where $\mathrm{N}_{\text {out }}$ and $\mathrm{N}_{\text {in }}$ are the signals inside and outside the detail respectively and $\mathrm{A}$ is the area of the detail [1]. The Mean Glandular Dose (MGD) (4) is given by equation 4 where $\mathrm{N}_{0}$ is the entrance signal, $(\mu / \rho)_{\text {air }}$ is the mass attenuation coefficient of air, E is the energy of the X-ray and $g$ is a conversion factor converting entrance dose to mean glandular dose ${ }^{12}$.

$$
\begin{aligned}
S N R & =\frac{\sqrt{A}\left|N_{\text {out }}-N_{\text {in }}\right|}{\sqrt{N_{\text {out }}}} \\
M G D & =\left(N_{0}\left(\frac{\mu}{\rho}\right)_{\text {air }} E\right) g
\end{aligned}
$$

The dose conversion factors are dependent on breast thickness equivalence (table 1$)^{13}$. The equation for the Half Value Layer (HVL) is given in equation 5 where $\mu$ is the attenuation coefficient of aluminium ${ }^{14}$.

Table 1. The equivalent breast thickness for a range of Perspex thicknesses.

\begin{tabular}{|c|c|}
\hline Perspex thickness $(\mathrm{mm})$ & Equivalent breast thickness $(\mathrm{mm})$ \\
\hline 30 & 32 \\
\hline 40 & 45 \\
\hline 50 & 60 \\
\hline 60 & 75 \\
\hline 70 & 90 \\
\hline
\end{tabular}

$$
H V L=\frac{0.693}{\mu}
$$

Equations 2-5 can be combined in order to calculate the IQF in terms of X-ray energy. This can be used to find the maximum IQF for different breast equivalent thicknesses.

\subsection{Image acquisition and processing}

The test object was imaged for a total $4 \mathrm{~cm}$ of Perspex. The imaging parameters were chosen using the calculations of the IQF in order to find the ideal energy for this thickness.

Images were acquired using a "step and shoot" method. Each image is laminar in shape due to the shape of the monochromatic beam. The final image is produced by combining the strips into one image. The width of each strip is dependent on the angle used. A small additional width is added to each image as an overlap region in order to reduce any artefacts that could occur at the joins between images.

A MATLAB routine is used to combine the strips. The strips of image are aligned and the overlap regions averaged. Flat-field corrections are applied to remove any detector related artefacts. Then the image can be windowed over a user specified energy range. 


\section{RESULTS}

\subsection{IQF energy data}

Using the theory presented in section 2.2, graphs were plotted of the IQF for a range of X-ray energies for three different Perspex thicknesses. This was done for two low contrast details, Nylon and wax (figure 3). The maximum achievable IQF is given by the maximum point on each of the graphs. This is given in table 2 along with the corresponding angle between monochromator and beam
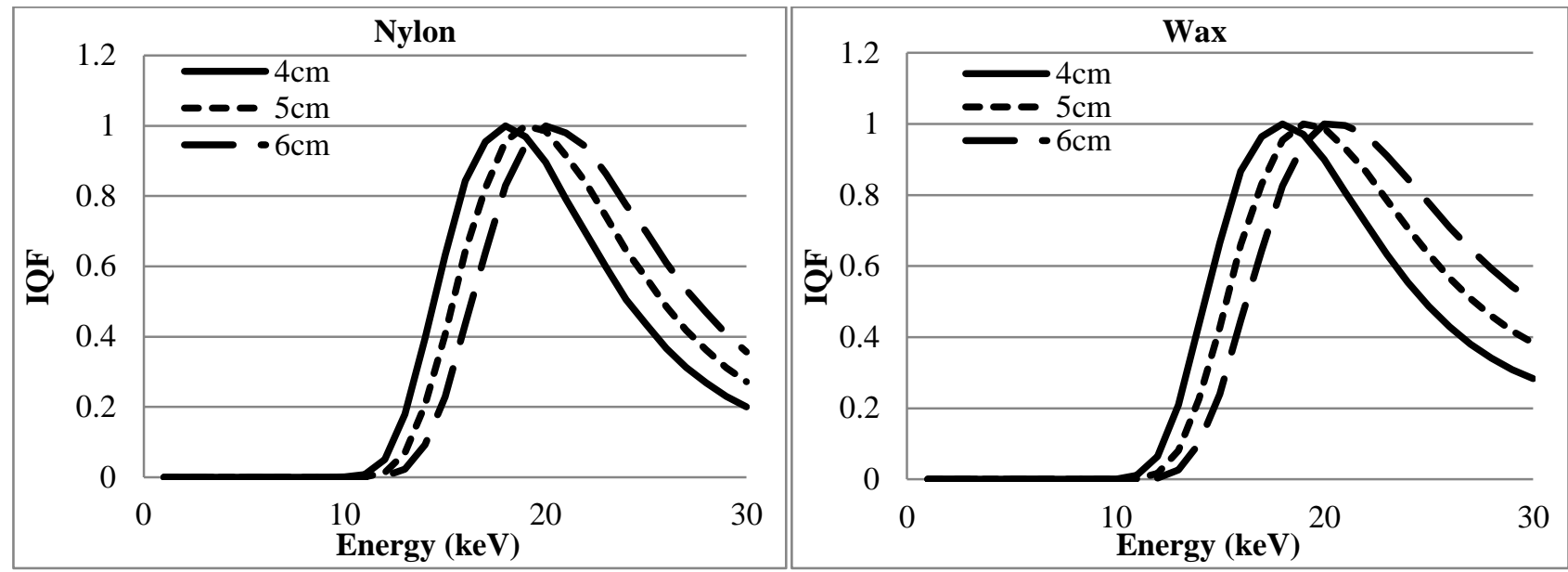

Figure 3. The graphs of IQF against X-ray energy for the Nylon and Wax details. Each were plotted for 4, 5 and $6 \mathrm{~cm}$ of Perspex.

The ideal energies that will be used to image the test object are shown in table 2 . The energy does not vary significantly between the Nylon and Wax details.

Table 2. The ideal energy and the equivalent crystal angle for the three different Perspex thicknesses. The data is for Nylon and Wax.

\begin{tabular}{|c|c|c|c|c|}
\hline & \multicolumn{2}{|c|}{ Nylon } & \multicolumn{2}{c|}{ Wax } \\
\hline Perspex thickness $(\mathrm{cm})$ & Energy $(\mathrm{keV})$ & Crystal angle $\left(^{\circ}\right)$ & Energy $(\mathrm{keV})$ & Crystal angle $\left(^{\circ}\right)$ \\
\hline 4 & 17.9 & 4.0 & 18.0 & 4.0 \\
\hline 5 & 19.2 & 3.8 & 19.2 & 3.8 \\
\hline 6 & 20.3 & 3.5 & 20.8 & 3.3 \\
\hline
\end{tabular}

\subsection{Monochromatic imaging data}

Monochromatic images were acquired of a $4 \mathrm{~cm}$ thick test object using an $18 \mathrm{keV}$ energy peak. Each strip of image was $3 \mathrm{~mm}$ in height corresponding to 12 pixels on the detector with an extra 2 pixels of overlap on either side of the image. The normalised energy spectrum for images taken with and without the $4 \mathrm{~cm}$ Perspex test object is shown in figure 4 . A greater low energy component can be seen when imaging the Perspex; this is the effect Compton-scattering has on the energy spectrum. The monochromatic energy peak is indicated at $18 \mathrm{keV}$. This spectrum was used to window the image around the monochromatic peak and the point at which this was done is indicated on the graph.

The image of the test object is shown in figure 5 with the scatter component removed. Contrast measurements were made on the scatter free and the full spectrum images; these values are stated in table 3.

The contrast values show a consistent improvement of around $20 \%$ when the scatter component of the image is removed. 


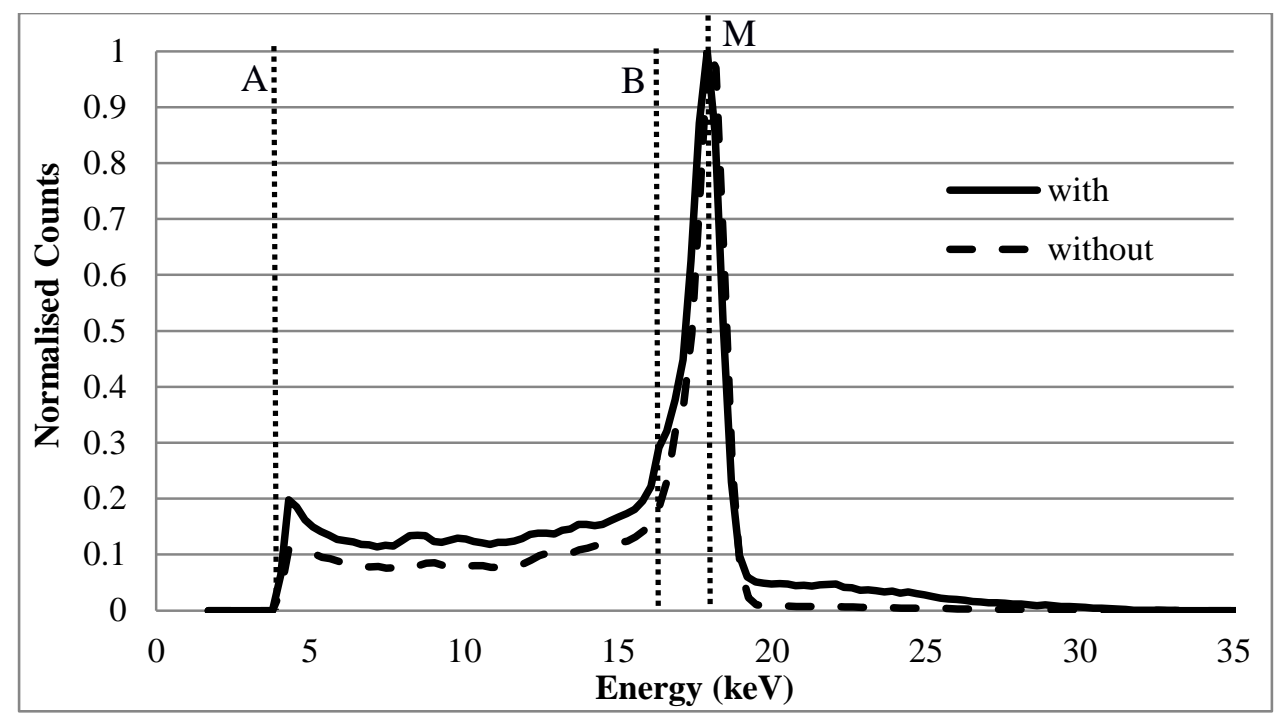

Figure 4. The normalised energy spectrum used to image the test object. This is shown both with and without the $4 \mathrm{~cm}$ thick Perspex test object. Point M indicates the monochromatic peak at $18 \mathrm{keV}$. Point A (4 keV) indicates the lower limit for the full energy spectrum image. Point B $(16 \mathrm{keV})$ indicates the lower limit for the windowed energy spectrum. The upper limit for both was the end of the spectrum at $35 \mathrm{keV}$.

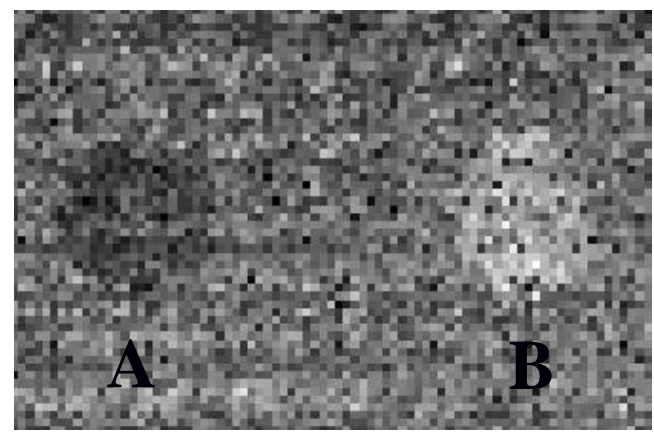

Figure 5. An image showing the test object after the scatter has been removed as shown in figure 4. A indicates the Nylon detail and $\mathrm{B}$ indicates the wax detail.

Table 3. The contrast measurements of the image both before and after windowing of the energy spectrum.

\begin{tabular}{|c|c|c|c|}
\hline Detail & Full energy spectrum image & Scatter free image & Percentage difference \\
\hline Nylon & $0.049 \pm 0.004$ & $0.062 \pm 0.004$ & $20 \%$ \\
\hline Wax & $0.054 \pm 0.003$ & $0.067 \pm 0.003$ & $19 \%$ \\
\hline
\end{tabular}

\section{CONCLUSIONS}

Results from a setup that produces scatter free images using a quasi-monochromatic X-ray beam coupled with a spectroscopic detector have been presented. It has been shown that the scatter can be removed from images by windowing around the monochromatic peak. Once scatter had been removed the contrast increased by $20 \%$.

Future work will address contrast improvement for a range of thicknesses and comparisons of the image quality/dose trade-off with conventional mammography.

\section{ACKNOWLEDGEMENTS}

The authors would like to thank EPSRC (Grant number: EP/H046577/1) for funding the project, HEXITEC and the University of Surrey. 


\section{REFERENCES}

[1] J T. Bushberg et al, [The essential physics of medical imaging], Lippincott Williams \& Wilkins (2012).

[2] R L Van Metter et al, [Handbook of Medical Imaging, Volume 1. Physics and Psychophysics], SPIE press (2000).

[3] C M Ronckers et al, "Radiation and breast cancer: a review of current evidence”, Breast Cancer Res (2005) 7:21-32

[4] S.Webb, [The physics of medical imaging], Bristol ; Philadelphia : Hilger (2012)

[5] P. Sprawls, [The physical principles of medical imaging], Aspen Publishers, (1993)

[6] "Highly Ordered Pyrolytic Graphite" <http://www.2spi.com/catalog/new/hopgsub.php> (21st November 2013)

[7]_S N. Ahmed, [Physics and Engineering of radiation detection], San Diego; London: Academic Press (2007)

[8] M C.Veale et al, "X-ray microbeam characterisation of a small pixel spectroscopic CdTe detector”, (2012) JINST 7 P07017.

[9] M D. Wilson et al, "Multiple Module Pixellated CdTe Spectroscopic X-Ray Detector”, IEEE Transactions on nuclear science (2013) 60, 21197 - 1200

[10] K C Young et al, "Dose and image quality in mammography with an automatic beam quality system", The British Journal of Radiology (1996) 69:822, 555-562

[11] Horst Aichinger et al, [Radiation Exposure and Image Quality in X-Ray Diagnostic Radiology: Physical Principles and Clinical Applications], Springer Science \& Business Media, (2013)

[12] D R Dance et al, "Monte-Carlo calculation of conversion factors for the estimation of mean glandular breast dose", (1990) Phys. Med. Biol. 351211

[13] Ulrich Bick et al, [Digital Mammography], Springer Science \& Business Media, (2010)

[14] Jerrold T. Bushberg, [The Essential Physics of Medical Imaging], Lippincott Williams \& Wilkins, (2002) 